\section{REVIEW ARTICLE}

Jeffrey M. Drazen, M.D., Editor

\title{
Muco-Obstructive Lung Diseases
}

\author{
Richard C. Boucher, M.D.
}

\section{A} SPECTRUM OF LUNG DISEASES THAT AFFECT THE AIRWAYS, INCLUDING chronic obstructive pulmonary disease (COPD), cystic fibrosis, primary ciliary dyskinesia, and non-cystic fibrosis bronchiectasis, can be characterized as muco-obstructive diseases. ${ }^{1-4}$ These diseases have the clinical features of cough, sputum production, and episodic exacerbations that are often associated with a diagnosis of chronic bronchitis. ${ }^{5}$ However, neither "chronic bronchitis" nor "hypersecretory diseases" ${ }^{\circ}$ adequately describes the diffuse mucus obstruction, airway-wall ectasia, chronic inflammation, and bacterial infection that are typical of these conditions; therefore, "muco-obstructive" may be a preferred descriptive term. Although asthma can also be associated with diffuse airway mucus obstruction, ${ }^{7}$ its distinct pathophysiological mechanisms preclude discussion in this grouping. ${ }^{8}$

The pathogenesis of muco-obstruction of the airways is depicted in Figure 1A. In healthy persons, a well-hydrated mucus layer is transported rapidly (at a rate of approximately $50 \mu \mathrm{m}$ per second) from the distal airways toward the trachea. In muco-obstructive diseases, epithelial defects in ion-fluid transport, mucin secretion, or a combination of these lead to hyperconcentrated (dehydrated) mucus, failed mucus transport, and mucus adhesion to airway surfaces. Mucus that is accumulated in the trachea can be expectorated by cough as phlegm or sputum. ${ }^{11}$ Mucus in the small airways cannot be cleared by cough and accumulates, forming the nidus for airflow obstruction, infection, and inflammation. ${ }^{11}$

BIOCHEMICAL AND BIOPHYSICAL PROPERTIES OF MUCUS

RELATIVE TO AIRWAY FUNCTION

Human airway mucus is a hydrogel composed of approximately $98 \%$ water, $0.9 \%$ salt, $0.8 \%$ globular proteins, and $0.3 \%$ high-molecular-weight mucin polymers. ${ }^{12}$ The hydration (concentration) status of mucus is measured as the wet-to-dry content of mucus (i.e., the percentage of a given volume that consists of nonvolatile solids) or as the mucin concentration determined by light-scattering techniques. ${ }^{9,11,13}$ The correlation between the two measurements (in healthy persons or those with muco-obstructive disease) is high, which allows both measures to be used to describe this mucus property. ${ }^{9}$

The two major synthesized and secreted respiratory mucins, MUC5B and MUC5AC, are physically very large, spanning 0.2 to $10 \mu \mathrm{m}$ in length for single polymers. The secreted mucin polymers interweave to form mesh-like gels with mesh sizes that are concentration dependent (i.e., higher concentrations are associated with smaller mesh sizes). MUC5B and MUC5AC share many features, including their multimeric organization and high carbohydrate content (approximately $75 \%$ of total weight) (Fig. 1B). ${ }^{14}$ There are, however, major differences in the
From the Marsico Lung Institute, University of North Carolina at Chapel Hill, Chapel Hill. Address reprint requests to Dr. Boucher at the Marsico Lung Institute, University of North Carolina at Chapel Hill, 7008 Marsico Hall, Chapel Hill, NC 27599, or at richard_boucher@med.unc.edu.

N Engl J Med 2019;380:1941-53. DOI: 10.1056/NEJMra1813799 Copyright (c) 2019 Massachusetts Medical Society. 


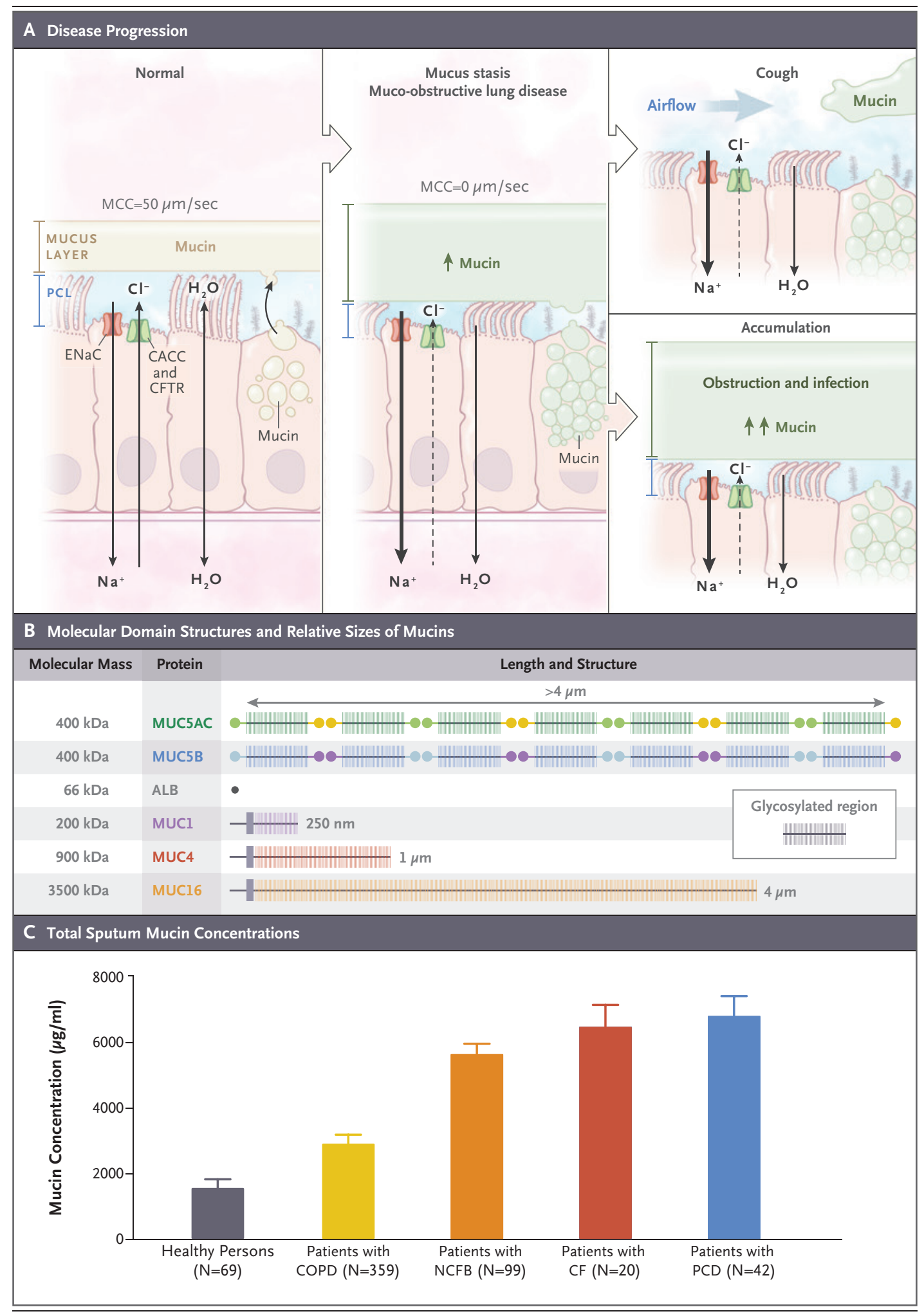


Figure 1 (facing page). Muco-Obstructive Disease Pathogenesis, Mucin Species, and Total Mucin Concentrations in Health and Disease.

Panel A shows the progression from normal to mucoobstructed airways. In healthy persons (left), wellbalanced epithelial sodium $\left(\mathrm{Na}^{+}\right)$absorption and secretion of chloride anions $\left(\mathrm{Cl}^{-}\right)$hydrates airway surfaces and promotes efficient mucociliary clearance (MCC). In persons with muco-obstructive lung disease (middle), an imbalance of ion transport coupled with mucin hypersecretion increases mucin concentrations in the mucus layer, osmotically compresses the periciliary layer (PCL), and abolishes MCC. The adherent mucus may be expelled as sputum by cough (upper right). Mucus that cannot be expelled by cough accumulates, concentrates, obstructs airflow, and becomes the nidus for infection (lower right). CFTR denotes cystic fibrosis transmembrane conductance regulator, and $\mathrm{ENaC}$ the epithelial sodium channel. Panel B shows domain structures and relative sizes of the secreted mucins (MUC5AC and MUC5B) and tethered mucins (MUCl, MUC4, and MUC16). For reference, the globular protein albumin (ALB) is shown. The secreted mucins are composed of monomers with $\mathrm{N}$ terminals (MUC5AC, green; MUC5B, light blue), glycosylated domains, and $C$ terminals (MUC5AC, yellow; MUC5B, purple). Both the $\mathrm{C}-\mathrm{C}$ terminal dimers and $\mathrm{N}-\mathrm{N}$ terminal multimers are linked by $\mathrm{S}-\mathrm{S}$ bonds. The glycosylated domains contain sugar side chains from 2 to 15 sugar molecules in length, often terminally capped with sialic acid or sulfate, which gives mucins a negative charge. The glycosylation domains provide mucin hydration, reflecting the avidity of sugar molecules for water, and a combinatorial library of binding sites capable of trapping most inhaled materials with a low but sufficient binding affinity to mediate clearance. The tethered mucins have cytoplasmic $\mathrm{N}$-terminal (dark gray), transmembrane (light gray), and heavily glycosylated extracellular domains. For simplicity, unique domains and proteolytic cleavage sites are not shown. Panel $\mathrm{C}$ shows total sputum mucin concentrations in healthy persons and patients with chronic obstructive pulmonary disease (COPD), noncystic fibrosis bronchiectasis (NCFB), cystic fibrosis (CF), or primary ciliary dyskinesia (PCD). Sputum mucin concentrations were measured by means of high-performance liquid chromatography and refractometry. ${ }^{9,10}$ $T$ bars indicate standard errors of the mean.

regulation of transcription and probably the function of MUC5B and MUC5AC. Data from studies in mice indicate that Muc5b is required for basal mucociliary transport, whereas Muc5ac reacts to external stresses, features that are likely to be mimicked in humans. Recent data indicate that MUC5B is by concentration the dominant mucin (10:1 over MUC5AC) in normal human lower airways. ${ }^{10}$

The physical properties of mucus can be predicted from polymer physics ${ }^{15}$ that describe not only well-studied mucus viscoelastic properties but also other key biophysical properties, including mucus osmotic pressure, adhesion, cohesion, and friction. ${ }^{11,13,15}$ The viscoelastic and osmotic properties of mucus, as with other polymer gels, scale to the third power, or higher, of concentration. This feature of mucus accounts for the observation that relatively small changes in concentration have profound effects on the biophysical and transport properties of mucus for example, higher concentrations dramatically decrease transportability. ${ }^{13,16}$ In addition, the properties of mucus gels can be modified by conditions that create inter-mucin polymer interactions with relatively long bond half-lives, producing "sticky" polymer gels whose viscous properties can scale to the eighth power of concentration. ${ }^{13,15}$

\section{MUCIN CONCENTRATIONS IN MUCO-} OBSTRUCTIVE LUNG DISEASES

Mucin concentrations are abnormally raised in each of the four muco-obstructive diseases COPD, cystic fibrosis, primary ciliary dyskinesia, and non-cystic fibrosis bronchiectasis - and these findings are consistent with a role for elevated mucin concentrations in the pathogenesis of muco-obstructive disease (Fig. 1C). Although MUC5AC levels are elevated and may uniquely modify mucus properties, MUC5B is the dominant mucin by concentration in these diseases. ${ }^{10}$

INTEGRATED CILIA AND COUGH-

DEPENDENT MUCUS CLEARANCE

The classic views of the mucus transport system have undergone substantial revision at both macroscopic and microscopic length scales during the past decade. ${ }^{13,17}$

\section{MACROSCOPIC - INTRAPULMONARY MUCIN SOURCES}

Mucus lines all airway surfaces as it moves toward the throat through ciliary activity. Tradi- 


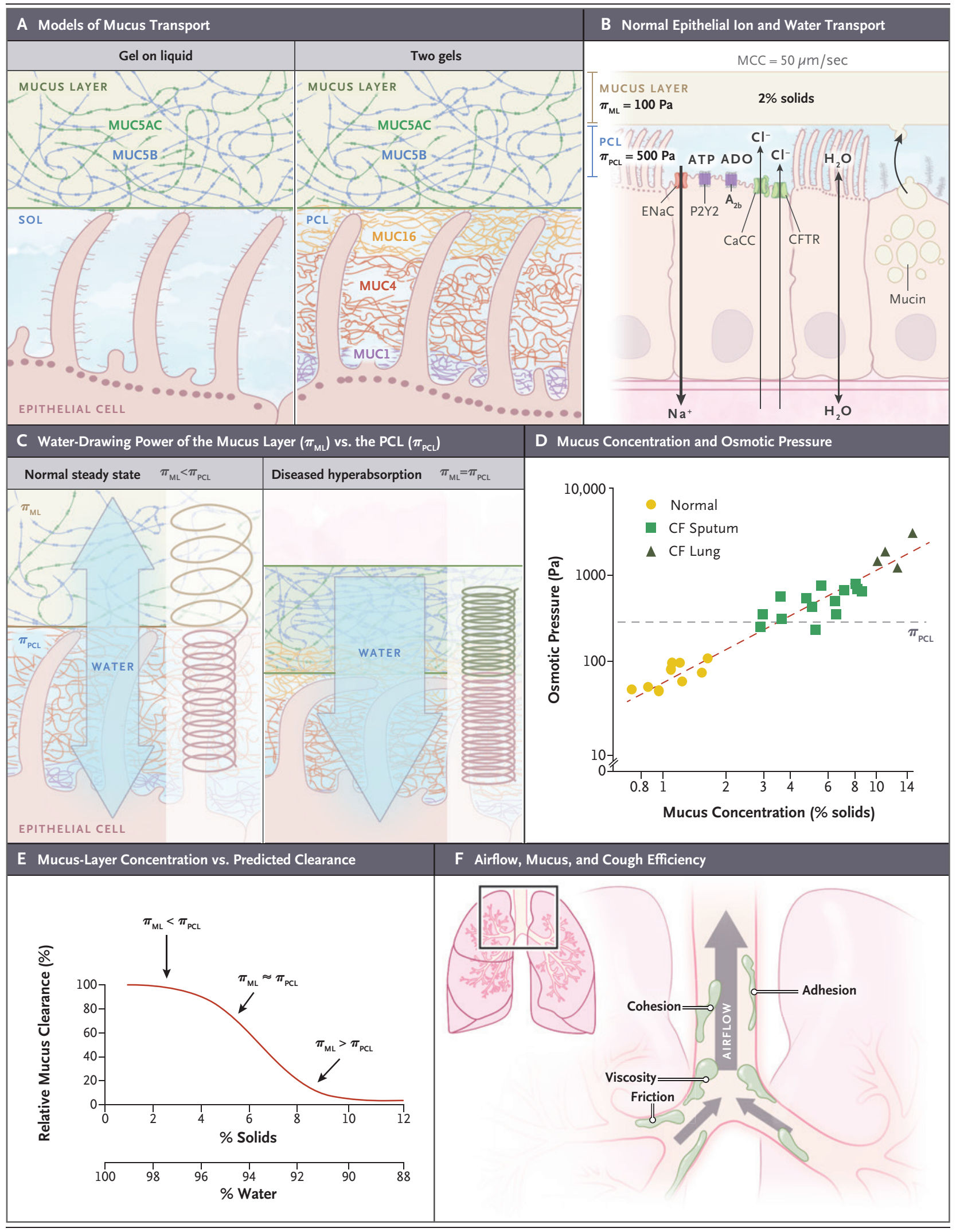


Figure 2 (facing page). Two-Gel Model of Mucus Transport. Panel A, left, shows the classic "gel-on-liquid" model showing a mucus layer (MUC5AC and MUC5B) and a "sol" layer as liquid surrounding cilia. Panel $A$, right, shows the "twogel" model in which the mucus layer remains the same, but a periciliary layer (gel) is formed by tethered macromolecules, including $\mathrm{MUCl}, \mathrm{MUC4}$, and $\mathrm{MUCl6}$. Panel B shows epithelial ion and water transport in normal human airway epithelia. The percentage of solids in the normal mucus layer and osmotic pressures $(\pi)$ of the normal mucus layer $(\pi \mathrm{ML})$ and $\mathrm{PCL}(\pi \mathrm{PCL})$ in pascals $(\mathrm{Pa})$ are noted. An $\mathrm{ENaC}$ in the apical membrane mediates sodium and liquid absorption..$^{21}$ In parallel, the epithelium secretes chloride and bicarbonate anions through CFTR and calciumactivated chloride channels ( $\mathrm{CaCC}){ }^{21}$ The balance between active sodium absorption and secretion of chloride and bicarbonate anions, and hence fluid flow, is regulated in part by lumenal concentrations of extracellular ATP, interacting through $\mathrm{P} 2 \mathrm{Y} 2$ receptors, and adenosine (ADO), interacting through $\mathrm{A}_{2 b}$ receptors. ${ }^{22}$ Panel $\mathrm{C}$ describes the relative waterdrawing powers of the mucus layer $\left(\pi_{\mathrm{ML}}\right)$ and $\mathrm{PCL}\left(\pi_{\mathrm{PCL}}\right)$ interfaced to epithelial cell-mediated fluid absorption or secretion (blue arrows). The length of the Hookean springs (to the right of the blue arrows) denotes the height of the PCL (purple) or mucus layer (green), and the spring diameter is inversely proportional to osmotic pressure. In a normal state (left), $\pi_{\mathrm{ML}}$ is lower than $\pi_{\mathrm{PCL}}$, represented by a green spring $\left(\pi_{\mathrm{ML}}\right)$ with a diameter larger than the purple spring $(\pi \mathrm{PCL})$. In a dehydrated state (right), persistent or abnormal absorption initially removes fluid from the lower-osmoticpressure mucus layer but ultimately coordinately removes fluid from both the mucus layer and the PCL. The osmotic pressures of both layers are increased and equalized (smaller spring diameters) and volume depleted (springs shortened). This state osmotically compresses the cilia and produces mucus stasis. Panel $D$ shows the relationship between mucus concentration (i.e., percent solids) and osmotic pressure $(\mathrm{Pa}) .{ }^{9}$ Samples of mucus from healthy persons, sputum from patients with cystic fibrosis, and mucus obtained from excised lungs of patients with cystic fibrosis are shown. Osmotic pressures in the mucus layer that are higher than the basal osmotic pressure of the PCL (black dashed line) are predicted to produce collapse of the PCL and slow MCC. ${ }^{13}$ Panel E shows MCC as a function of mucus-layer concentration predicted from the ratio of $\pi \mathrm{ML}$ to $\pi \mathrm{PCL}$. At normal mucus hydration ( $2 \%$ solids and $98 \%$ water), $\pi_{\mathrm{ML}}$ is below basal $\pi \mathrm{PCL}$ values, the PCL is well hydrated, and mucus transport is efficient. With modest mucus dehydration ( 3 to $4 \%$ solids and 96 to $97 \%$ water), $\pi_{\mathrm{ML}}$ slightly exceeds $\pi_{\mathrm{PCL}}$, modest compression of the PCL results, and MCC slows. When mucus dehydration is severe ( 7 to $10 \%$ solids and 90 to $93 \%$ water), the mucus layer osmotically compresses cilia, producing mucus stasis. Panel $\mathrm{F}$ shows interactions of airflow with mucus biophysical properties relevant to cough efficiency. In more distal airways (below bifurcation), reduced airflows (smaller arrows) can only slide mucus along airway surfaces, a movement limited by friction between mucus and cell surfaces. At airway bifurcations, mucus remodels as it enters the proximal airway, which has a reduced aggregate surface area, a process limited by mucus viscosity. ${ }^{11}$ In large airways (e.g., the trachea), the higher airflow (larger arrow) fractures intramucus cohesive forces, adhesive forces between mucus and the cell surface, or both to expel mucus as sputum. ${ }^{23}$ tionally, the superficial epithelia that line large (bronchial) and small (bronchiolar) airways were believed to secrete MUC5AC, whereas large-airway submucosal glands were thought to secrete MUC5B. Data from mouse models and human studies have led to a reformulation of this view. ${ }^{18,19}$ Under baseline conditions, the superficial epithelia of both human large and small airways secrete MUC5B. Airway MUC5B secretion is supplemented by MUC5B secretion from the glands that may be in part tonic but probably can be greatly increased with cough. ${ }^{20} \mathrm{~A}$ variety of pulmonary stresses trigger superficial epithelial MUC5AC expression. ${ }^{12}$ Recent research suggests that MUC5AC secretion is superimposed on superficial airway secretory (club) cells that are competent for basal MUC5B secretion. ${ }^{18}$

\section{MICROSCOPIC - MUCUS LAYER-CILIA TOPOGRAPHY}

The classic description of mucociliary clearance envisioned the movement of a mucus (gel) layer over a periciliary watery (liquid) layer, also called the "sol" layer. ${ }^{17}$ This description has been replaced by one depicting the mucociliary apparatus as comprising two gels: a mucus layer and a periciliary layer (Fig. 2A). ${ }^{13}$ The periciliary layer was identified as a dense gel composed of glycopolymers, including the MUC1, MUC4, and MUC16 mucins, tethered to epithelial-cell surfaces and cilia. An important concept that emerged from this formulation is not only that the mucociliary-clearance apparatus is composed of two polymer hydrogels but also that they compete for hydration. The force that mediates polymer-gel hydration - that is, the waterdrawing power - is described as the osmotic pressure of a hydrogel. Experimental measurements revealed that the osmotic pressure of the normal mucus layer consisting of $2 \%$ solids is approximately $100 \mathrm{~Pa},{ }^{9,13}$ whereas the periciliary layer is more concentrated, with an osmotic pressure of approximately $500 \mathrm{~Pa}$ (Fig. 2B). ${ }^{13}$ The higher osmotic pressure of the periciliary layer in healthy persons ensures that it is well hydrated, providing appropriate lubrication for ciliary activity and transport of the overlying mucus layer. ${ }^{13}$

\section{INTEGRATION OF EPITHELIAL ION TRANSPORT}

\section{AND MUCUS HYDRATION}

The two-gel model allows for quantitative predictions with respect to interactions between 


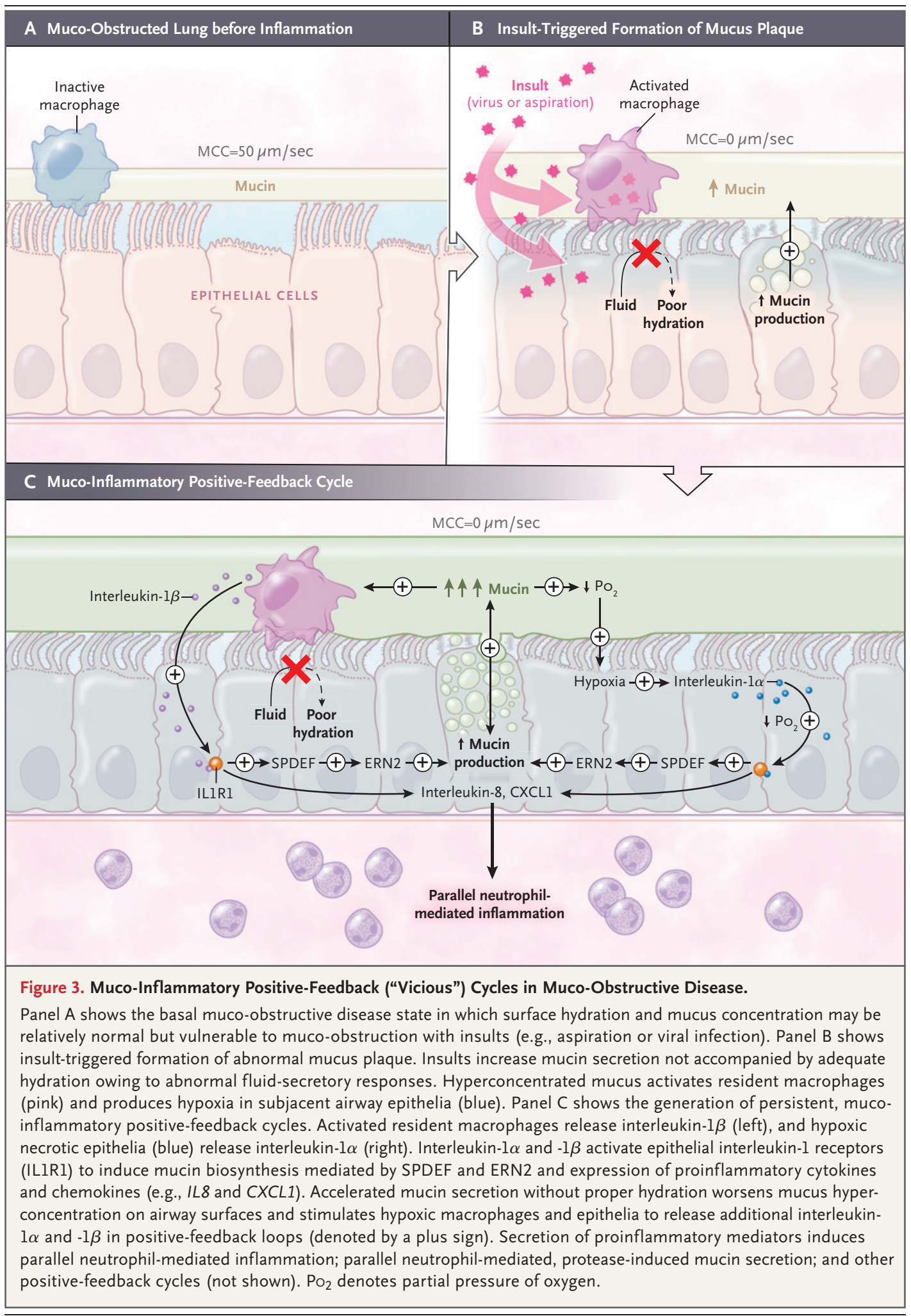


mucus-layer concentration (hydration) and the efficiency of mucociliary transport. These predictions are best understood from analyses of how the mucus and periciliary layers respond to fluid transport by the underlying airway epithelium.

In healthy persons, airway epithelia can secrete or absorb ions and water and probably do both simultaneously. ${ }^{21}$ Maintenance of normal airway-surface hydration is provided by cilial mechanosensing of mucus-layer concentration, regulated release of ATP by ciliated cells onto the airway surface, and fine-tuning of ion-transport and fluid-transport rates through purinoceptor activation (Fig. 2B), ${ }^{8,24}$ In the well-hydrated normal state ( $2 \%$ solids and $98 \%$ water), the mucus layer acts as a fluid buffer to ensure efficient mucus transport (Fig. 2C; and Fig. S1 in the Supplementary Appendix, available with the text of this article at NEJM.org). However, in muco-obstructive disease, abnormal epithelial fluid absorption depletes the airway surface of fluid, increases mucus concentrations, and raises osmotic pressures in the mucus layer to levels exceeding those in the periciliary layer (Fig. 2C). Moderately hyperconcentrated mucus that is associated with disease compresses cilia and slows mucus transport, whereas severe hyperconcentration flattens cilia and produces mucus stasis and adhesion. Thus, the two-gel formulation predicts mucociliary transport rates from mucus concentrations (Fig. 2D and 2E) and explains why relatively small changes in hydration status ( $2 \%$ vs. $8 \%$ solids [i.e., $98 \%$ vs. $92 \%$ water]) produce disease.

\section{COUGH - BACKUP MECHANISM RECRUITED TO CLEAR ADHERENT MUCUS}

When cilial-dependent mucus clearance fails, cough becomes the backup clearance mechanism. ${ }^{23}$ It is the combined failure of cilialdependent and cough-dependent clearance that produces muco-obstructive disease. Like cilialdependent mucus transport, mucus concentration is a key determinant for all mucus properties associated with cough-dependent transport, including friction, viscosity, cohesion, and adhesion (Fig. 2F, and Fig. S2 in the Supplementary Appendix). ${ }^{11}$
PATHOGENESIS

OF MUCO-OBSTRUCTION

An important feature of muco-obstructive lung diseases is disease heterogeneity - that is, there are regions of the lung that are normal and other regions within the same lung that are severely diseased. Bronchiectasis is often a prominent finding in computed tomography (CT) of the lung. However, a common feature that unifies muco-obstructive diseases is the early manifestation of disease in small airways (bronchioles), as evidenced by pathological examination, microcomputed tomography, and pulmonary-function studies.

The airways are defended against infection both by mechanical (mucus) clearance and by antimicrobial proteins and peptides that are secreted into mucus by airway epithelial cells. ${ }^{25,26}$ The ability of antimicrobial molecules and antibodies to suppress bacterial-infection replication is short lived, probably on the order of hours. ${ }^{26}$ Consequently, a kinetic "horse race" is manifest in the lung, pitting the rate of acquisition of bacterial resistance to endogenous antimicrobial suppression against the rate of mechanical (mucus) bacterial clearance. However, slowing of clearance alone is probably not sufficient to produce disease. It appears that the mucus layer needs to come to a stop, with the formation of mucus plaques and plugs within airway lumens, to produce muco-obstructive disease. Studies in animal models have shown that the full spectrum of muco-obstructive diseases, including airflow obstruction, inflammation, and intermittent infection, are observed with mucus concentration-dependent formation of plaques and plugs but not isolated defects in mucociliary clearance. ${ }^{27}$

Mucus plaque or plug formation in the airways reflects an increase in mucin secretion, often stimulated by viral infections or aspiration, coupled with poor epithelial hydration of newly secreted mucins (Fig. 3A and 3B). On formation of hyperconcentrated, static mucus plaques, a bistable positive-feedback ("vicious") muco-inflammatory cycle is initiated (Fig. 3C). ${ }^{28,29}$ Ultimately, severe or persistent muco-obstruction, typically associated with elevated airway levels of neutrophil elastase, drives the progres- 
sion from bronchitic to bronchiectatic or bronchiolectatic lesions typical of these diseases. ${ }^{30,31}$

Bacterial infection is a common feature of muco-obstructive lung diseases. ${ }^{2-4,32}$ Data from patients with cystic fibrosis or other mucoobstructive diseases indicate that the major sites of infection in muco-obstructive lung diseases are intralumenal mucus plaques and plugs, not airway epithelial-cell surfaces. ${ }^{32}$ The limitation of oxygen diffusion through mucus plaques to underlying actively metabolizing epithelial cells produces regions of hypoxia within mucus plaques (Fig. 3). ${ }^{32,33}$ Analyses of microbiome data from patients with cystic fibrosis or COPD have identified oral anaerobes as the first bacterial pathogens in the muco-obstructed lung. ${ }^{34,35}$ These data suggest that a mouth-lung aspiration axis, in which oral anaerobes infect hypoxic mucus plaques, may be a unifying concept for early bacterial infection in most muco-obstructive diseases. ${ }^{35,36}$ Anaerobic bacteria can modify the hypoxic mucus environment to promote invasion by classic pathogens (e.g., Pseudomonas aeruginosa), which respond to mucus concentration-dependent tightening of mucus meshes to grow as aggregates or biofilms., ${ }^{42,33,37,38}$ Future studies are required to establish whether oral pharyngeal flora in sputum samples obtained from patients with muco-obstructive disease may, like classic pathogens, require treatment.

EXACERBATIONS

Exacerbations are typical of muco-obstructive diseases. An exacerbation is broadly defined as a change in the patient's perception of wellbeing, the seeking of health care, or a health care-implemented change in the patient's medical regimen..$^{39}$ In all muco-obstructive lung diseases, the overall rate of progression of disease severity (e.g., loss of lung function) is probably heavily influenced by the incidence and severity of acute exacerbations. Common to all mucoobstructive diseases, previous rates of exacerbation and gastric aspiration are predictors of future rates of exacerbation - that is, a patient with many past exacerbations is more likely to have future exacerbations than a patient without such a history. ${ }^{1}$

It is important to note that an exacerbation is superimposed on preexisting, heterogeneous muco-obstructive lung disease. Previous studies suggest that some exacerbations are caused by an intensification of disease in areas with preexisting disease, perhaps reflecting a change in bacteria through genetic drift or phenotypic responses to a local environment. ${ }^{40,41}$ However, the new physical findings that are associated with exacerbations suggest that many exacerbations are associated with the spread of disease to previously unaffected regions (Fig. S3 in the Supplementary Appendix). ${ }^{42}$ A common mechanism for spread may be gastric aspiration; this concept is consistent with data from patients with COPD, non-cystic fibrosis bronchiectasis, or cystic fibrosis. ${ }^{43,44}$ Perhaps the major trigger for spread is viruses aspirated from upper airways into the lung. A spread mechanism is consistent with data from patients with cystic fibrosis or noncystic fibrosis bronchiectasis showing that the microbiome changes little during exacerbations. ${ }^{34,45}$ Clinically, exacerbations must be treated vigorously to limit permanent loss of lung function.

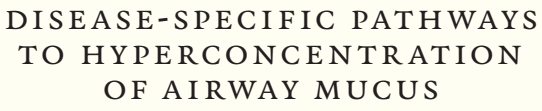

\section{CYSTIC FIBROSIS}

Cystic fibrosis is the most extensively studied disease with respect to the two-gel hypothesis describing mucus concentration-dependent disease. The abnormal concentration of mucus in the lungs of patients with cystic fibrosis reflects a primary abnormality of airway epithelial ion transport. ${ }^{4}$ In this disease, the airway epithelium is vulnerable to fluid hyperabsorption because of a defect in the secretion of chloride and bicarbonate anions mediated by the cystic fibrosis transmembrane conductance regulator (CFTR) and by an intact sodium absorptive path. Laboratory evidence suggests that cystic fibrosis can be triggered by viral infections that lead to unrestrained liquid absorption, mucus hyperconcentration, and the formation of mucus plaques and plugs (Fig. S4 in the Supplementary Appendix). ${ }^{22}$

Mucus hyperconcentration as a feature of cystic fibrosis was first documented in adults. Sputum measurements revealed higher total mucin concentrations (Fig. 1C) and a higher percentage of solids (Fig. S4 in the Supplemen- 
tary Appendix) in patients with cystic fibrosis than in healthy persons. ${ }^{9}$ Sputum osmotic pressures in healthy persons (approximately $100 \mathrm{~Pa}$ ) were below the osmotic pressure of the periciliary layer $(500 \mathrm{~Pa})$, as predicted,$^{13}$ whereas expectorated sputum samples from patients with cystic fibrosis had osmotic pressures slightly above basal periciliary-layer values $(\geq 500 \mathrm{~Pa})$ (Fig. 2D). The mucus extracted from the lungs of patients with cystic fibrosis at transplantation had even higher concentrations (10 to $15 \%$ solids) and osmotic pressures (>1000 Pa), findings consistent with a failure to clear (cough out) highly concentrated, adherent mucus., ${ }^{911}$ Optical coherence tomography of the upper airways in vivo of adults with cystic fibrosis revealed evidence of mucus hyperconcentration, slowed mucociliary clearance, and, as predicted by the two-gel hypothesis, a reduction in the height of the periciliary layer. ${ }^{46}$

Recent data regarding bronchoalveolar lavage from children with cystic fibrosis in the Australian Respiratory Early Surveillance Team for Cystic Fibrosis (AREST CF) cohort showed that a persistent increase in mucin concentration and inflammatory cells preceded bacterial infection in the lung. ${ }^{47}$ "Rough" (hyperconcentrated) MUC5B and MUC5AC mucus flakes, probably of smallairway origin, were also a feature of bronchoalveolar fluid from children with cystic fibrosis. ${ }^{48}$ Although failure of CFTR-dependent bicarbonate secretion might suggest a role for $\mathrm{pH}$ in early cystic fibrosis, transbronchoscopic measurements in the AREST CF cohort did not detect a significant difference in lower-airway $\mathrm{pH}$ between patients with cystic fibrosis and disease controls without cystic fibrosis. In addition, $\mathrm{pH}$ has less effect than concentration on the biophysical properties of mucus related to clearance or plaque formation..$^{11}$ Aspirated oral anaerobes appear to be the first bacterial pathogens in the lungs of patients with cystic fibrosis, followed by the classic gram-negative pathogens that probably accelerate the vicious cycle depicted in Figure 3 and loss of lung function.,44,50

\section{COPD}

COPD manifests as persistent airflow obstruction, most often in response to inhaled environmental agents. ${ }^{1,51}$ Hyperconcentrated mucus has been reported in lower-airway samples obtained from patients with COPD, and higher mucus concentrations are correlated with reduced in vivo rates of mucociliary clearance. ${ }^{16}$ Data from a large cohort study involving patients with COPD suggest that a major trigger of COPD, cigarette smoke, is associated with an increase in mucin concentration..$^{10}$ Furthermore, hyperconcentration of mucins in sputum was associated in this cohort with disease severity, as indexed by associations between increased mucin concentrations and greater airflow obstruction and higher exacerbation rates. ${ }^{10}$ Increased mucin concentrations were also associated with a subgroup of patients with early COPD (i.e., those with nearly normal lung function), who were shown to be at risk for rapid progression of their disease. $^{10}$

The airway epithelial defects that produce mucus hyperconcentration in COPD are complex. ${ }^{51}$ Exposure to cigarette smoke may produce abnormalities in CFTR-mediated secretion of chloride anions through oxidant-induced reduction of CFTR transcription rates and direct damage to CFTR protein in the apical membrane. ${ }^{52,53}$ Furthermore, increased extracellular nucleotide and nucleoside metabolism decreases extracellular ATP and adenosine levels, which suggests defective purinoceptor regulation of airway-surface hydration. ${ }^{16}$ These defects in epithelial ion and fluid transport (hydration) are amplified by cigarette smoke-induced hypersecretion of MUC5AC and MUC5B. ${ }^{6}$ Mucin hypersecretion may be particularly important in COPD because accelerated metabolism of co-released adenosine diphosphate, adenosine monophosphate, and adenosine, coupled with defective CFTR function, may limit paracrine adenosine-CFTR hydration responses that accompany mucin secretion. ${ }^{54}$

The mucin hyperconcentration that characterizes patients with COPD is perhaps the mildest example in the muco-obstructive disease grouping (Fig. 1C). Patients with COPD also tend to have the least severe muco-obstructive airway disease, particularly with respect to the lower incidence of bronchiectasis and pseudomonas infection (Fig. 2B)..$^{55,56}$

\section{PRIMARY CILIARY DYSKINESIA}

The pathogenesis of primary ciliary dyskinesia has typically been considered as solely a "motor problem" caused by genetic abnormalities in 
cilial shaft proteins, cilial beat frequency, and defective mucociliary clearance., ${ }^{3,57}$ However, sputum obtained from patients with primary ciliary dyskinesia also is abnormally hyperconcentrated, which suggests that a "second hit" contributes to the pathogenesis of the disease (Fig. 1C).$^{58}$ Studies that identified the mechanotransduction pathways linking mucus concentration with cilial strain-induced ATP release also showed that this signaling pathway was defective in patients with primary ciliary dyskinesia. ${ }^{24}$ This defect in ciliary sensing probably reduces the release of ATP onto airway surfaces, thereby decreasing fluid secretion and generating a hyperconcentrated mucus plaque or plug component to airflow obstruction.

The pulmonary phenotype of patients with primary ciliary dyskinesia, aside from the more basilar distribution of bronchiolectasis and bronchiectasis, is similar to that of cystic fibrosis but is shifted toward older ages with respect to disease severity-age relationships., ${ }^{3,59}$ Notable similarities to cystic fibrosis include the presence of anaerobes in early primary ciliary dyskinesia, the subsequent acquisition of classic muco-obstructive or bronchiectatic pathogens (e.g., staphylococcus, Haemophilus influenzae, and P. aeruginosa), and severe neutrophilic inflammation in the airways with raised DNA concentrations. ${ }^{3}$ It is not clear whether the milder disease phenotype of patients with primary ciliary dyskinesia reflects the retention of partial ciliary activity in many genotypes of the disease, the persistence of CFTR functions other than hydration in primary ciliary dyskinesia, or other factors.

\section{NON-CYSTIC FIBROSIS BRONCHIECTASIS}

Non-cystic fibrosis bronchiectasis is a phenotype defined by bronchiectasis in the absence of a monogenetic cause. ${ }^{60}$ Although non-cystic fibrosis bronchiectasis is now typically defined by dilated bronchi on CT, classic pathological studies have highlighted severe small-airway disease, including bronchiolectasis, mucus plugging, and inflammation, in this syndrome. ${ }^{61}$ Consistent with these pathological findings, non-cystic fibrosis bronchiectasis has the typical small-airway airflow impairment of muco-obstructive diseases. ${ }^{62}$

Non-cystic fibrosis bronchiectasis probably reflects an interaction between environmental stresses and genetic host-defense risk alleles, with bronchiectasis as a final common pathway. ${ }^{60}$ Genetic studies suggest that non-cystic fibrosis bronchiectasis is associated with a spectrum of genetic risks, including abnormal mucosal host defense, immune function, or connective tissue. ${ }^{60}$ The recent reports that sputum obtained from patients with non-cystic fibrosis bronchiectasis is hyperconcentrated suggest that an epithelial defect in the regulation of mucin hydration may contribute to disease pathogenesis (Fig. 1C). Currently, genetic studies have not linked ion-transport genes to non-cystic fibrosis bronchiectasis, and direct measurements have suggested that CFTR functions normally in patients with non-cystic fibrosis bronchiectasis without CFTR mutations. ${ }^{63}$

The microbiome of patients with non-cystic fibrosis bronchiectasis is typical of muco-obstructive diseases, characterized by polymicrobial oral anaerobic bacteria, staphylococcus, and H. influenzae, with approximately 15 to $20 \%$ of patients positive for P. aeruginosa. ${ }^{45,64}$ In this context, noncystic fibrosis bronchiectasis resembles early cystic fibrosis. ${ }^{50}$ Patients with non-cystic fibrosis bronchiectasis have an incidence of nontuberculous mycobacteria infection that may be as much as $60 \%$ higher than the incidence among patients with other muco-obstructive diseases; this finding indicates a potential role of immunedeficiency alleles in non-cystic fibrosis bronchiectasis. ${ }^{60,65}$

\section{AGNOSIS}

Disease-specific criteria assist in the diagnosis of each muco-obstructive lung disease: for cystic fibrosis, levels of chloride anions in sweat and genetic testing ${ }^{66}$; for COPD, exposure history and spirometry ${ }^{1}$; for primary ciliary dyskinesia, nasal nitric-oxide measurements, cilia waveform analyses, and genetic testing 3,57 ; and for noncystic fibrosis bronchiectasis, CT scanning. ${ }^{60}$ The tools to make a general diagnosis of mucoobstructive disease are also available. Patientrelated outcomes regarding the presence of cough, sputum production, and exacerbation frequency can be obtained from general respiratory questionnaires. ${ }^{5}$ Measures of sputum inflammatory cells and cytokines or chemokines aid in establishing the inflammatory component of airway muco-obstruction. 
Measures of mucus concentration (percent solids) and total mucin concentrations have also been explored as diagnostic tools. ${ }^{10}$ For example, mucin concentrations in sputum are significantly associated with chronic bronchitis symptoms defined by patient-related outcome data, and receiver-operating-characteristic curve analyses suggest that mucin concentration is a good biomarker for COPD. ${ }^{10}$

THERAPIES FOR MUCO-OBSTRUCTIVE DISEASES

Disease-specific therapies for one muco-obstructive disease, cystic fibrosis, are now available. Ivacaftor (VX-770) is a potentiator of residual CFTR function that has been approved for patients with cystic fibrosis with gating and some splicing CFTR mutations ${ }^{67}$ Ivacaftor provided a model for the development of therapies for mucoobstruction, as evidenced by impressive associations between improved airway-surface hydration in vitro, peripheral and central mucociliary clearance in vivo, and clinically relevant outcomes (e.g., forced expiratory volume in 1 second $\left.\left[\mathrm{FEV}_{1}\right]\right)$. For patients with cystic fibrosis who are homozygous for a deletion of phenylalanine 508 (F508del) in CFTR, ivacaftor-lumacaftor and tezacaftor-ivacaftor are available but offer more modest clinical benefit. Three-drug combinations of correctors and potentiators await approval but appear to be highly effective in patients with at least one F508del mutation (approximately 90\% of patients).

\section{HYDRATORS}

Perhaps the most direct approach in the treatment of muco-obstructive lung diseases is to reduce the concentration of pathologic mucus - that is, rehydrate it. The currently available approach to achieve this goal is inhalation of osmotically active aerosols (e.g., hypertonic saline or mannitol). ${ }^{68,69}$ In clinical development are modulators of ion transport that may redirect airway epithelial ion transport from net absorptive to secretory directions, providing a mechanism for epithelial restoration of airway-surface hydration. ${ }^{70}$

Hypertonic saline is the most widely tested inhaled osmotic agent in muco-obstructive lung diseases. The use of hypertonic saline is best described in cystic fibrosis, producing sustained increases in mucociliary clearance, increased $\mathrm{FEV}_{1}$, and reduced frequencies of exacerbation. ${ }^{69,71}$ Inhalation of hypertonic saline is often used in patients with non-cystic fibrosis bronchiectasis or primary ciliary dyskinesia, with some early evidence of efficacy, ${ }^{72}$ whereas inhalation of hypertonic saline appears to be safe in sputum induction in patients with COPD but is untested as yet therapeutically. ${ }^{73}$

\section{MUCOLYTICS}

Two recent observations suggest that attacking abnormal mesh and gel properties of mucus in addition to reducing concentration may be therapeutic. First, mucus viscosity is a key variable governing the mucus cohesive and adhesive properties relevant to cough efficiency, and reductions in viscosity with mucin S-S bond-reducing or surfactant agents decrease mucus adhesion and cohesion independent of concentration. ${ }^{11}$ Second, the mucus plaques or flakes that are recovered from young patients with cystic fibrosis during bronchoalveolar lavage are unable to swell and dissolve in excess solvent ${ }^{47}$ (i.e., they are "permanent" gels). Permanency can be conferred by the generation of bonds with a long half-life between mucin polymers that can reflect oxidant-induced inter-mucin S-S bonds, inflammatory protein-mediated "sticker" interactions between adjacent mucins, or both. ${ }^{74}$ Thus, therapies that are designed to reduce inter-mucin S-S bonds or block mucin-sticker interactions (e.g., with surfactants or glycopolymers) are being developed to improve both mucus swelling and cough clearance. ${ }^{8,75}$ Inhaled acetylcysteine has not proved to have the mucin-reductive activity required for a therapeutic effect. ${ }^{8}$ (Additional references for this article are listed in the Supplementary Appendix.)

\section{CONCLUSIONS}

Muco-obstructive diseases are characterized by mucus hyperconcentration. The four mucoobstructive diseases differ with respect to the epithelial abnormalities that produce mucus hyperconcentration but follow a final common path of mucus concentration-dependent formation of mucus plaques and plugs. Adherent mucus plaques may stimulate a positive-feedback muco- 
inflammatory cycle that renders mucus plaques permanent and damaging to airway walls. Therapies that are designed to rehydrate and restore mucous viscous or elastic properties are rational. The challenge is to deliver these therapies to the small airways, where mucus obstruction may be complete and the physics of aerosol-deposition efficiency may be poor. However, the concept that these diseases may be highly treatable emanates from studies involving patients with cystic fibrosis in whom potentiators have reversed mucus obstruction in both large and small airways. ${ }^{67}$

Disclosure forms provided by the author are available with the full text of this article at NEJM.org.
REFERENCES

1. Han MK, Agusti A, Calverley PM, et al. Chronic obstructive pulmonary disease phenotypes: the future of COPD. Am J Respir Crit Care Med 2010;182:598-604.

2. King PT, Holdsworth SR, Freezer NJ, Villanueva E, Holmes PW. Characterisation of the onset and presenting clinical features of adult bronchiectasis. Respir Med 2006;100:2183-9.

3. Knowles MR, Zariwala M, Leigh $M$. Primary ciliary dyskinesia. Clin Chest Med 2016;37:449-61.

4. Rowe SM, Miller S, Sorscher EJ. Cystic fibrosis. N Engl J Med 2005;352:19922001.

5. Jones PW, Forde Y. St George's respiratory questionnaire manual. London: St George's, University of London, 2009 (http://www.healthstatus.sgul.ac.uk/SGRQ _download/SGRQ\%20Manual\%20June\% 202009.pdf).

6. Rogers DF. Physiology of airway mucus secretion and pathophysiology of hypersecretion. Respir Care 2007;52:113446.

7. Dunican EM, Elicker BM, Gierada DS, et al. Mucus plugs in patients with asthma linked to eosinophilia and airflow obstruction. J Clin Invest 2018;128:997-1009. 8. Fahy JV, Dickey BF. Airway mucus function and dysfunction. N Engl J Med 2010;363:2233-47.

9. Henderson AG, Ehre C, Button B, et al. Cystic fibrosis airway secretions exhibit mucin hyperconcentration and increased osmotic pressure. J Clin Invest 2014;124: 3047-60.

10. Kesimer M, Ford AA, Ceppe A, et al. Airway mucin concentration as a marker of chronic bronchitis. N Engl J Med 2017; 377:911-22.

11. Button B, Goodell HP, Atieh E, et al. Roles of mucus adhesion and cohesion in cough clearance. Proc Natl Acad Sci U S A 2018;115:12501-6.

12. Rose MC, Voynow JA. Respiratory tract mucin genes and mucin glycoproteins in health and disease. Physiol Rev 2006;86: 245-78.

13. Button B, Cai LH, Ehre C, et al. A periciliary brush promotes the lung health by separating the mucus layer from airway epithelia. Science 2012;337:937-41.

14. Thornton DJ, Rousseau K, McGuckin MA. Structure and function of the poly- meric mucins in airways mucus. Annu Rev Physiol 2008;70:459-86.

15. Rubinstein M, Colby RH. Polymer physics. Oxford, United Kingdom: Oxford University Press, 2003.

16. Anderson WH, Coakley RD, Button B, et al. The relationship of mucus concentration (hydration) to mucus osmotic pressure and transport in chronic bronchitis. Am J Respir Crit Care Med 2015;192:182 90.

17. Lucas A, Douglas LC. Principles under lying ciliary activity in the respiratory tract. Arch Otolaryngol 1934;20:518-41.

18. Okuda K, Chen G, Subramani DB, et al. Localization of secretory mucins MUC5AC and MUC5B in normal/healthy human airways. Am J Respir Crit Care Med 2019;199:715-27.

19. Zhu Y, Ehre C, Abdullah LH, et al Munc13-2-/- baseline secretion defect reveals source of oligomeric mucins in mouse airways. J Physiol 2008;586:1977-92.

20. Widdicombe JH, Wine JJ. Airway gland structure and function. Physiol Rev 2015; 95:1241-319.

21. Boucher RC. Human airway ion transport: part one. Am J Respir Crit Care Med 1994;150:271-81.

22. Tarran R, Button B, Picher M, et al Normal and cystic fibrosis airway surface liquid homeostasis: the effects of phasic shear stress and viral infections. J Biol Chem 2005;280:35751-9.

23. Leith DE. Cough. Phys Ther 1968;48. 439-47.

24. Button B, Okada SF, Frederick CB, Thelin WR, Boucher RC. Mechanosensitive ATP release maintains proper mucus hydration of airways. Sci Signal 2013;6: ra46.

25. Cole AM, Liao HI, Stuchlik O, Tilan J, Pohl J, Ganz T. Cationic polypeptides are required for antibacterial activity of human airway fluid. J Immunol 2002;169: 6985-91.

26. Cole AM, Dewan P, Ganz T. Innate antimicrobial activity of nasal secretions. Infect Immun 1999;67:3267-75.

27. Livraghi-Butrico A, Grubb BR, Wilkinson KJ, et al. Contribution of mucus concentration and secreted mucins Muc5ac and Muc5b to the pathogenesis of mucoobstructive lung disease. Mucosal Immunol 2017;10:395-407.
28. Cole PJ. Inflammation: a two-edged sword - the model of bronchiectasis. Eur J Respir Dis Suppl 1986;147:6-15.

29. Tyson JJ, Chen KC, Novak B. Sniffers, buzzers, toggles and blinkers: dynamics of regulatory and signaling pathways in the cell. Curr Opin Cell Biol 2003;15:221-31.

30. Chalmers JD, Moffitt KL, SuarezCuartin G, et al. Neutrophil elastase activity is associated with exacerbations and lung function decline in bronchiectasis. Am J Respir Crit Care Med 2017;195:138493.

31. Sly PD, Gangell CL, Chen L, et al. Risk factors for bronchiectasis in children with cystic fibrosis. N Engl J Med 2013;368: 1963-70.

32. Worlitzsch D, Tarran R, Ulrich $M$, et al. Effects of reduced mucus oxygen concentration in airway Pseudomonas infections of cystic fibrosis patients. J Clin Invest 2002;109:317-25.

33. Cowley ES, Kopf SH, LaRiviere A, Ziebis W, Newman DK. Pediatric cystic fibrosis sputum can be chemically dynamic, anoxic, and extremely reduced due to hydrogen sulfide formation. MBio 2015; 6(4):e00767.

34. Tunney MM, Field TR, Moriarty TF, et al. Detection of anaerobic bacteria in high numbers in sputum from patients with cystic fibrosis. Am J Respir Crit Care Med 2008;177:995-1001.

35. Sze MA, Hogg JC, Sin DD. Bacterial microbiome of lungs in COPD. Int J Chron Obstruct Pulmon Dis 2014;9:229-38.

36. Segal LN, Clemente JC, Tsay JC, et al. Enrichment of the lung microbiome with oral taxa is associated with lung inflammation of a Th17 phenotype. Nat Microbiol 2016;1:16031.

37. Flynn JM, Niccum D, Dunitz JM, Hunter RC. Evidence and role for bacterial mucin degradation in cystic fibrosis airway disease. PLoS Pathog 2016;12(8): e1005846.

38. Matsui H, Wagner VE, Hill DB, et al. A physical linkage between cystic fibrosis airway surface dehydration and Pseudomonas aeruginosa biofilms. Proc Natl Acad Sci U S A 2006;103:18131-6.

39. Rosenfeld M, Emerson J, WilliamsWarren J, et al. Defining a pulmonary exacerbation in cystic fibrosis. J Pediatr 2001;139:359-65. 
40. Sethi S, Maloney J, Grove L, Wrona C, Berenson CS. Airway inflammation and bronchial bacterial colonization in chronic obstructive pulmonary disease. Am J Respir Crit Care Med 2006;173:991-8.

41. Goss $\mathrm{CH}$, Burns JL. Exacerbations in cystic fibrosis. 1: Epidemiology and pathogenesis. Thorax 2007;62:360-7.

42. Boucher RC. On the pathogenesis of acute exacerbations of mucoobstructive lung diseases. Ann Am Thorac Soc 2015; 12:Suppl 2:S160-S163.

43. Lee AL, Button BM, Denehy L, et al. Proximal and distal gastro-oesophageal reflux in chronic obstructive pulmonary disease and bronchiectasis. Respirology 2014;19:211-7.

44. Brodlie M, Aseeri A, Lordan JL, et al. Bile acid aspiration in people with cystic fibrosis before and after lung transplantation. Eur Respir J 2015;46:1820-3.

45. Cox MJ, Turek EM, Hennessy C, et al. Longitudinal assessment of sputum microbiome by sequencing of the $16 \mathrm{~S}$ rRNA gene in non-cystic fibrosis bronchiectasis patients. PLoS One 2017;12(2):e0170622.

46. Leung HM, Birket S, Hyun C, et al. The study of functional microanatomy of CF airways using clinical intranasal microOCT imaging. Pediatr Pulmonol 2018;53: Suppl 2:174. abstract.

47. Esther CR, Muhlebach MS, Ehre C, et al. Mucus accumulation in the lungs precedes structural changes and infection in children with cystic fibrosis. Sci Transl Med 2019;11(486):eaav3488.

48. Simel DL, Mastin JP, Pratt PC, et al. Scanning electron microscopic study of the airways in normal children and in patients with cystic fibrosis and other lung diseases. Pediatr Pathol 1984;2:4764.

49. Abdullah LH, Coakley R, Webster MJ, et al. Mucin production and hydration responses to mucopurulent materials in normal versus cystic fibrosis airway epithelia. Am J Respir Crit Care Med 2018;197: 481-91.

50. Muhlebach MS, Zorn BT, Esther CR, et al. Initial acquisition and succession of the cystic fibrosis lung microbiome is associated with disease progression in infants and preschool children. PLoS Pathog 2018; 14(1):e1006798.

51. Hogg JC, Paré PD, Hackett TL. The contribution of small airway obstruction to the pathogenesis of chronic obstructive pulmonary disease. Physiol Rev 2017;97: 529-52.

52. Clunes LA, Davies CM, Coakley RD, et al. Cigarette smoke exposure induces CFTR internalization and insolubility, leading to airway surface liquid dehydration. FASEB J 2012;26:533-45.

53. Cantin AM, Bilodeau G, Ouellet C, Liao J, Hanrahan JW. Oxidant stress suppresses CFTR expression. Am J Physiol Cell Physiol 2006;290:C262-C270.

54. Kreda SM, Seminario-Vidal L, van Heusden CA, et al. Receptor-promoted exocytosis of airway epithelial mucin granules containing a spectrum of adenine nucleotides. J Physiol 2010;588:2255-67. 55. Martinez-Garcia MA, Miravitlles M. Bronchiectasis in COPD patients: more than a comorbidity? Int J Chron Obstruct Pulmon Dis 2017;12:1401-11.

56. Murphy TF, Brauer AL, Eschberger K, et al. Pseudomonas aeruginosa in chronic obstructive pulmonary disease. Am J Respir Crit Care Med 2008;177:853-60.

57. Horani A, Ferkol TW, Dutcher SK, Brody SL. Genetics and biology of primary ciliary dyskinesia. Paediatr Respir Rev 2016;18:18-24.

58. Bush A, Payne D, Pike S, Jenkins G, Henke MO, Rubin BK. Mucus properties in children with primary ciliary dyskinesia: comparison with cystic fibrosis. Chest 2006;129:118-23.

59. Maglione M, Bush A, Nielsen KG, et al. Multicenter analysis of body mass index, lung function, and sputum microbiology in primary ciliary dyskinesia. Pediatr Pulmonol 2014;49:1243-50.

60. Flume PA, Chalmers JD, Olivier KN. Advances in bronchiectasis: endotyping, genetics, microbiome, and disease heterogeneity. Lancet 2018;392:880-90.

61. Whitwell F. A study of the pathology and pathogenesis of bronchiectasis. Thorax 1952;7:213-39.

62. Roberts HR, Wells AU, Milne DG, et al. Airflow obstruction in bronchiectasis: correlation between computed tomography features and pulmonary function tests. Thorax 2000;55:198-204.

63. Bienvenu T, Sermet-Gaudelus I, Burgel PR, et al. Cystic fibrosis transmembrane conductance regulator channel dysfunction in non-cystic fibrosis bronchiectasis. Am J Respir Crit Care Med 2010;181:107884.

64. Serisier DJ, Martin ML, McGuckin MA, et al. Effect of long-term, low-dose erythromycin on pulmonary exacerbations among patients with non-cystic fibrosis bronchiectasis: the BLESS randomized controlled trial. JAMA 2013;309:1260-7.

65. Aksamit TR, O'Donnell AE, Barker A, et al. Adult patients with bronchiectasis: a first look at the US Bronchiectasis Research Registry. Chest 2017;151:982-92.

66. Farrell PM, White TB, Ren CL, et al. Diagnosis of cystic fibrosis: consensus guidelines from the Cystic Fibrosis Foundation. J Pediatr 2017;181:Suppl:S4-S15.e1. 67. Ramsey BW, Davies J, McElvaney NG, et al. A CFTR potentiator in patients with cystic fibrosis and the G551D mutation. N Engl J Med 2011;365:1663-72.

68. Anderson SD, Daviskas E, Brannan JD, Chan HK. Repurposing excipients as active inhalation agents: the mannitol story. Adv Drug Deliv Rev 2018;133:4556.

69. Donaldson SH, Bennett WD, Zeman KL, Knowles MR, Tarran R, Boucher RC. Mucus clearance and lung function in cystic fibrosis with hypertonic saline. $\mathrm{N}$ Engl J Med 2006;354:241-50.

70. Shei RJ, Peabody JE, Kaza N, Rowe SM. The epithelial sodium channel $(\mathrm{ENaC})$ as a therapeutic target for cystic fibrosis. Curr Opin Pharmacol 2018;43:152-65.

71. Elkins MR, Robinson M, Rose BR, et al. A controlled trial of long-term inhaled hypertonic saline in patients with cystic fibrosis. N Engl J Med 2006;354:229-40.

72. Paff T, Daniels JM, Weersink EJ, Lutter R, Vonk Noordegraaf A, Haarman EG. A randomised controlled trial on the effect of inhaled hypertonic saline on quality of life in primary ciliary dyskinesia. Eur Respir J 2017;49:49.

73. Fujimoto K, Yasuo M, Urushibata K, Hanaoka M, Koizumi T, Kubo K. Airway inflammation during stable and acutely exacerbated chronic obstructive pulmonary disease. Eur Respir J 2005;25:640-6. 74. Yuan S, Hollinger M, LachowiczScroggins ME, et al. Oxidation increases mucin polymer cross-links to stiffen airway mucus gels. Sci Transl Med 2015;7: 276ra27.

75. Tarrant BJ, Le Maitre C, Romero L, et al. Mucoactive agents for chronic, non-cystic fibrosis lung disease: a systematic review and meta-analysis. Respirology 2017;22: 1084-92.

Copyright (c) 2019 Massachusetts Medical Society. 